\title{
Calibrating the experimental measurement of psychological attributes
}

Dominik R Bach ${ }^{1-3 *}$, Filip Melinščak ${ }^{3}$, Stephen M Fleming ${ }^{1,2,4}$, Manuel C Voelkle ${ }^{5,6}$

${ }^{1}$ Wellcome Centre for Human Neuroimaging, University College London, United Kingdom

${ }^{2}$ Max Planck UCL Centre for Computational Psychiatry and Aging Research, University College London, United Kingdom

${ }^{3}$ Computational Psychiatry Research, Department of Psychiatry, Psychotherapy, and Psychosomatics, Psychiatric Hospital, University of Zurich, Switzerland

${ }^{4}$ Department of Experimental Psychology, University College London, United Kingdom

${ }^{5}$ Psychological Research Methods, Humboldt University, Berlin, Germany

${ }^{6}$ Center for Lifespan Psychology, Max Planck Institute for Human Development, Berlin, Germany

*Corresponding author (d.bach@ucl.ac.uk)

This article has been published as

Bach DR, Melinščak F, Fleming SM, Voelkle M (2020). Calibrating the experimental measurement of psychological attributes. Nature Human Behaviour, 4, 1229-1235.

http://dx.doi.org/10.1038/s41562-020-00976-8

The journal's copyright restrictions do not permit posting the final author version on this pre-print server.

A freely accessible author version can be found here:

https://discovery.ucl.ac.uk/id/eprint/10116203/ 